\title{
THE INFLUENCE OF FORWARD SPEED AND NONLINEARITIES ON THE DYNAMIC BEHAVIOUR OF A CONTAINER SHIP IN REGULAR WAVES
}

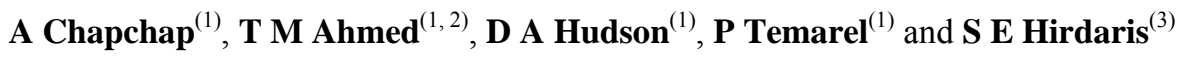 \\ (1) Ship Science, School of Engineering Sciences, University of Southampton, UK \\ (2) Department of Naval Architecture \& Marine Engineering, Alexandria University, Egypt \\ (3) Strategic Research Group, Lloyd's Register Marine Business, UK
}

\section{SUMMARY}

The aim of this paper is to compare the heave and pitch motions for the S175 containership, travelling in head regular waves, obtained from frequency domain linear and time domain partly nonlinear potential flow analyses. The frequency domain methods comprise the pulsating and the translating, pulsating Green's function methods, with the relevant source distribution over the mean wetted surface of the hull. The time domain method uses the radiation and diffraction potentials related to the mean wetted surface, implemented using Impulse Response Functions (IRF), whilst the incident wave and restoring actions are evaluated on the instantaneous wetted surface. The calculations are carried out for a range of Froude numbers, and in the case of the partly nonlinear method for different wave steepness values. Comparisons are made with available experimental measurements. The discussion focuses on the necessity for a nonlinear approach for predicting the radiation potential and the possible numerical methods for its formulation.

\section{NOMENCLATURE}

$\mathrm{a}$

$A_{\mathrm{ij}}$

$\boldsymbol{B}_{\mathrm{ij}}$

$C_{\mathrm{ij}}$

$F_{\mathrm{i}}$

Fn

$g$

$I_{\text {yy }}$

$k$

$L$

$m$ *

$m_{\mathrm{q}}{ }^{*}$

$m_{\mathrm{w}}$
$M_{\mathrm{ij}}$

$\tilde{M}_{\mathrm{q}}, \tilde{M}_{\dot{\mathrm{q}}}$

$\tilde{M}_{\mathrm{w}}, \tilde{M}_{\dot{\mathrm{w}}}$

$q, \dot{q}$

$t$

$w, \dot{w}$

$U$

$z_{\mathrm{q}}{ }^{*}$

$z_{\mathrm{W}}{ }^{*}$
Wave amplitude (m)

Element of hydrodynamic added mass matrix in equilibrium axes

Element of hydrodynamic damping matrix in equilibrium axes

Element of hydrostatic restoring matrix in equilibrium axes

Element of wave excitation vector in equilibrium axes

Froude number

Acceleration due to gravity $\left(\mathrm{m} \mathrm{s}^{-2}\right)$

Pitch moment of inertia $\left(\mathrm{kg} \mathrm{m}^{2}\right)$

Wave number (deep water, $k=\omega^{2} / g$ )

Length between perpendiculars $(\mathrm{m})$

Mass (kg)

Pitch-pitch IRF in body axes $\left(\mathrm{kg} \mathrm{m}^{2} \mathrm{~s}^{-2}\right)$

Pitch-heave IRF in body axes $\left(\mathrm{kg} \mathrm{m} \mathrm{s}^{-2}\right)$

Element of mass matrix in equilibrium axes

Pitch-pitch oscillatory coefficients for velocity and acceleration in body axes $\left(\mathrm{kg} \mathrm{m}^{2} \mathrm{~s}^{-1}, \mathrm{~kg} \mathrm{~m}^{2}\right)$

Pitch-heave oscillatory coefficients for velocity and acceleration in body axes (kg m s$\left.{ }^{-1}, \mathrm{~kg} \mathrm{~m}\right)$

Pitch velocity and acceleration in body axes ( $\left.\mathrm{rad} \mathrm{s}^{-1}, \operatorname{rad~s}^{-2}\right)$

Time (s)

Heave velocity and acceleration in body axes $\left(\mathrm{m} \mathrm{s}^{-1}, \mathrm{~m} \mathrm{~s}^{-2}\right)$

Forward speed of ship $\left(\mathrm{m} \mathrm{s}^{-1}\right)$

Heave-pitch IRF in body axes $\left(\mathrm{kg} \mathrm{m} \mathrm{s}^{-2}\right)$

Heave-heave IRF in body axes $\left(\mathrm{kg} \mathrm{s}^{-2}\right)$

$\begin{array}{ll}\widetilde{Z}_{\mathrm{q}}, \widetilde{Z}_{\dot{\mathrm{q}}} & \begin{array}{l}\text { Heave-pitch oscillatory coefficients for } \\ \text { velocity and acceleration in body axes } \\ \left(\mathrm{kg} \mathrm{m} \mathrm{s}^{-1}, \mathrm{~kg} \mathrm{~m}\right)\end{array} \\ \widetilde{Z}_{\mathrm{w}}, \widetilde{Z}_{\dot{\mathrm{w}}} & \begin{array}{l}\text { Heave-heave oscillatory coefficients } \\ \text { for velocity and acceleration in body } \\ \text { axes }\left(\mathrm{kg} \mathrm{s}^{-1}, \mathrm{~kg}\right)\end{array} \\ \theta & \text { Euler pitch angle (rad) } \\ \lambda & \text { Wave length (m) } \\ \xi_{\mathrm{j}} & \text { Element of motion vector in } \\ \bar{\varphi} & \text { equilibrium axes } \\ \varphi_{0} & \text { Steady velocity potential } \\ \varphi_{7} & \text { Incident wave potential } \\ \varphi_{\mathrm{j}} & \text { Diffracted wave potential } \\ \Phi & \text { Radiation potential } \\ \omega & \text { Total velocity potential } \\ \omega_{\mathrm{e}} & \left.\text { Wave frequency (rad s }{ }^{-1}\right)\end{array}$

\section{INTRODUCTION}

The development of the hydrodynamic theory of linear seakeeping for three-dimensional bodies commenced in the 1970s. Its main feature is to estimate the hydrodynamic coefficients (e.g. added mass and damping) and, for a given encounter frequency, use these values to calculate the ship motions in a seaway by solving a system of linear second order differential equations. In linear theory, several simplifications can be made enabling the total velocity potential to be expressed as a sum of different contributions. Each of these contributions has a specific physical meaning associated with well defined hydrodynamic actions. The main goal of linear frequency domain theory is to estimate the 
radiation potential which together with the incident wave potential are used to calculate the hydrodynamic coefficients and the exciting forces, the latter including diffraction effects.

The radiation potential problem is associated with the solution of the boundary value problem, which is derived from an application of Green's second identity to Laplace's equation and a suitable form of Green's function. The numerical problem is then formulated by discretising the boundary of the domain in order to distribute singularities over it. The solution of the boundary value problem provides the strengths of these singularities and, hence, the velocity potential.

A number of choices are available for the Green's function which dictates the extent of the domain discretisation and the boundary conditions to be satisfied. For instance, Inglis \& Price [1] used the pulsating source Green's function, which satisfies the far field (radiation) boundary condition and the linearised free surface boundary condition. This approach, therefore, requires only discretisation of the hull wetted surface. On the other hand, Sclavounos et al [2] used the simplest and most flexible form of Green's function to carry out a linear seakeeping analysis by means of the Rankine panel method. This approach requires discretisation of the free surface, in addition to the hull wetted surface, in order to satisfy an appropriate free surface condition. In addition the far field boundary condition needs to be satisfied through appropriate numerical schemes. Incorporating the effects of forward speed in the linear frequency domain analysis can also be accomplished by a suitable choice of Green's function. In this context, Inglis \& Price [3, 4] used a translating, pulsating Green's function in order to incorporate the effects of the perturbations of the flow due to steady forward motion of the hull on the radiation potential.

In seakeeping analysis, carried out using potential flow, the so called geometric nonlinear effects, in general, have three main sources of origin, namely the variation of the instantaneous wetted surface of the hull, higher order hydrodynamic actions and nonlinear free surface boundary condition. In order to tackle the sources of the nonlinearities, the assumptions involved in linear frequency domain analysis, such as a linearised free surface, small wave amplitudes and harmonic motion, need to be relaxed. This led to the development of a range of time domain potential flow methods with varying complexity depending on the nonlinear effects incorporated and the modelling of the free surface [5]. For example, Lin and Yue [6] used the time domain Green's function method, satisfying the exact body boundary condition on the instantaneous free surface and the linearised free surface condition on the flat free surface. Lin et al [7] extended this method to allow use of large wave amplitudes. Their approximation used the local free surface elevation to transform the hull geometry into a computational domain with a deformed body and a flat free surface.

Bailey et al [8] used an alternative approach for estimating hydrodynamic actions which, still within the bounds of linear theory, allows for fluid memory effects to be incorporated in the modelling of ship motions through the use of convolution integrals. This methodology implies that impulse response functions (IRFs) are available for the hydrodynamic diffraction and radiation forces. In fact, the IRFs are calculated based on the results of a linear frequency domain potential flow analysis. This type of model is usually called a partly nonlinear model, because it accounts exactly for non linear hydrostatics and wave exciting forces (FroudeKrylov), whilst the hydrodynamic radiation and diffraction effects are based on the mean wetted surface. The partly nonlinear model developed by Bailey et al [8] can be formulated either using the equilibrium axes, traditionally used for seakeeping, or the body (fixed) axes, traditionally used for manoeuvring. This model was extended and applied to various ship types by Ballard et al [9].

In this paper heave and pitch motions are investigated for the $\mathrm{S} 175$ containership travelling in head regular waves, for a range of forward speeds using linear and partly nonlinear methods. Pulsating and translating, pulsating source distributions over the mean wetted surface are used for the linear case [1, 4]. The partly nonlinear method developed by Bailey et al and Ballard et al $[8,9]$ is also applied, based on IRFs obtained from pulsating source hydrodynamic data, using a range of wave amplitudes or wave steepness values. Comparisons are made between the responses predicted by the different numerical methods and available experimental measurements by O'Dea et al [10]. The discussion focuses on possible ways of implementing fully nonlinear analysis to predict ship motions.

\section{LINEAR METHOD}

In this section a brief description of the main features of linear frequency domain theory is given. A more detailed description can be found in the literature [1]. The aim of linear frequency domain analysis is to solve Laplace's equation in the fluid domain, subject to the so called linearised boundary conditions, namely a boundary value problem. Once the boundary value problem is solved its solution is then used to estimate the hydrodynamic coefficients and the exciting forces acting on the ship. Subsequently the equations of motion, for each encounter frequency, are solved yielding transfer functions, or Response Amplitude Operators (RAOs) over the whole frequency range.

It is customary in this approach to decouple the velocity potential into two parts namely, steady and unsteady. In addition, the unsteady potential is also decomposed into 
components relating to incident wave excitation, diffraction and radiation. Furthermore, if the ship is in the presence of plane progressive waves, both fluid and rigid body motions can be considered to be time harmonic [11]. Hence, the total velocity potential can be written in the following form:

$$
\Phi(x, t)=U \bar{\varphi}+\mathfrak{R}\left[\exp \left(i \omega_{\mathrm{e}} t\right)\left(\varphi_{0}+\varphi_{7}+\sum_{\mathrm{j}=1}^{6} \xi_{\mathrm{j}} \varphi_{\mathrm{j}}\right)\right]
$$

In equation 1 , the components $\bar{\varphi}, \varphi_{0}$ and $\varphi_{7}$ refer to steady, incident wave and diffraction potentials, respectively. The steady potential is assumed to be independent of time. The components $\varphi_{\mathrm{j}}(\mathrm{j}=1$ to 6$)$ are the radiation potentials in surge, sway, heave, roll, pitch and yaw respectively, and $\xi_{\mathrm{j}}$ is the corresponding displacement in each of these modes. In equation $1 x$ denotes the equilibrium axis along the ship, with the equilibrium axis system typically situated at the mean water line with its origin aligned with the longitudinal position of the center of gravity, $z$ pointing upwards and $y$ to port. $U$ denotes forward speed and $\omega_{\mathrm{e}}$ the encounter frequency, evaluated in deep water.

For the linear problem in the frequency domain simplified boundary conditions can be obtained. For the incident wave and diffraction potentials the impervious boundary condition on the hull can be applied, namely:

$$
\frac{\partial\left(\varphi_{0}+\varphi_{7}\right)}{\partial n}=0 \quad \text { on } \bar{S}
$$

where $\bar{S}$ denotes the mean wetted surface of the hull. For the radiation contribution the boundary condition physically states that the normal velocity of the fluid is the same as the normal velocity of the hull on its (mean) wetted surface. Its treatment is more subtle because, in the presence of forward speed, it accounts for contributions not only of the radiation potentials but also the steady potential. It is in order to simplify the treatment of the radiation boundary condition that some simplifications in the form of the steady flow are made. Neglecting second order terms the steady flow velocity, in the equilibrium axis system, is expressed as $\boldsymbol{W}=U(\bar{\varphi}-x)$. The boundary condition for the radiation potentials then becomes [12]

$$
\frac{\partial \varphi_{\mathrm{j}}}{\partial n}=i \omega_{\mathrm{e}} n_{\mathrm{j}}+U m_{\mathrm{j}} \quad \text { on } \bar{S}
$$

where $n_{\mathrm{j}}$ denote the components of the normal vector and the terms $m_{\mathrm{j}}$ involve the influence of the steady flow [13]. If it is assumed that the perturbation of the flow due to steady forward motion can be neglected, then the $m_{\mathrm{j}}$ terms can be further simplified to $m_{\mathrm{j}}=0$ for $\mathrm{j}=1,2,3,4$ and $m_{5}=n_{3}$, $m_{6}=-n_{2}$ by uncoupling steady and unsteady flow effects, namely $\boldsymbol{W}=(-U, 0,0)$. Ahmed et al [14] investigated the influence of steady potential on the body boundary condition given by equation 3 , using both pulsating and translating, pulsating source formulations, and the subsequent hydrodynamic coefficients. Under these considerations, the linearised free surface boundary condition to be satisfied by the unsteady potentials is given by $[1,13]$

$\frac{\partial^{2} \varphi}{\partial t^{2}}-2 U \frac{\partial^{2} \varphi}{\partial x \partial t}+U^{2} \frac{\partial^{2} \varphi}{\partial x^{2}}+g \frac{\partial \varphi}{\partial z}=0 \quad$ on $z=0$

Equation 4 further simplifies for simple harmonic variation with time, with the pulsating source satisfying the zero forward speed and the translating, pulsating source the forward speed dependent free surface condition, respectively [13]. The remaining far field boundary condition is satisfied by both the pulsating and translating, pulsating Green's functions; hence, the boundary value problem is well posed.

Once the incident wave potential is given and the radiation and diffraction potentials are known one can estimate exciting forces and hydrodynamic coefficients at each encounter frequency. Therefore, the equations of motion for the ship in regular waves can be written as:

$$
\begin{array}{r}
\sum_{\mathrm{j}=1}^{6} \xi_{\mathrm{j}}\left[-\omega_{\mathrm{e}}^{2}\left(M_{\mathrm{ij}}+A_{\mathrm{ij}}\right)+i \omega_{\mathrm{e}} B_{\mathrm{ij}}+C_{\mathrm{ij}}\right]=\mathrm{a} F_{\mathrm{i}} \\
\text { for } \mathrm{i}=1,2, \ldots 6
\end{array} .
$$

Equation 5 is a system of six simultaneous linear equations which are solved for the ship motions $\xi_{\mathrm{j}}$. The coefficients $M_{\mathrm{ij}}$ refer to the mass and inertia properties of the ship. $A_{\mathrm{ij}}$ and $B_{\mathrm{ij}}$ are hydrodynamic added mass and damping coefficients obtained from the radiation potentials [1]. $C_{\mathrm{ij}}$ are the hydrostatic restoring coefficients and $\mathrm{a}$ is the wave amplitude of the regular wave. The quantities of interest in the present analysis are the heave and pitch motions, namely $\xi_{3}$ and $\xi_{5}$.

\section{PARTLY NONLINEAR METHOD}

In this section a summary of the main features of the partly nonlinear method is provided $[8,9]$. The main goal is not to explain the method in detail, but rather describe the basic equations and link the impulse response functions to the estimation of the coefficients for the system of differential equations to be solved.

In this paper when using the partly nonlinear method the ship motions are referenced to the body (fixed) axes. The body axes comprise an upright right handed coordinate axes $C x y z$ with the origin $C$ at the centre of gravity of the hull, and $C x z$ in its longitudinal plane of symmetry.

In the case of symmetric motions it can be shown that heave and pitch accelerations, $\dot{w}$ and $\dot{q}$, can be written as a function of the heave and pitch velocities, $w$ and $q$, 
the displacement of the ship centre of gravity, $z_{\mathrm{CG}}$, the Euler pitch angle, $\theta$ and time $t$. That is to say:

$$
\left[\begin{array}{c}
\dot{w}(t) \\
\dot{q}(t)
\end{array}\right]=\boldsymbol{M}^{-1}\left[\begin{array}{c}
\mathrm{f}_{\mathrm{w}}\left(w, q, z_{C G}, \theta, t\right) \\
\mathrm{f}_{\mathrm{q}}\left(w, q, z_{C G}, \theta, t\right)
\end{array}\right] .
$$

In this equation matrix $\boldsymbol{M}$ contains the ship mass $m$ and pitch moment of inertia $I_{\mathrm{yy}}$, as well the infinite frequency value of the acceleration oscillatory coefficients, e.g. $\widetilde{Z}_{\dot{\mathrm{w}}}$. That is to say:

$$
\boldsymbol{M}=\left[\begin{array}{cc}
m-\widetilde{Z}_{\dot{\mathrm{w}}}(\infty) & -\widetilde{Z}_{\dot{\mathrm{q}}}(\infty) \\
-\tilde{M}_{\dot{\mathrm{w}}}(\infty) & \mathrm{I}_{\mathrm{yy}}-\tilde{M}_{\dot{\mathrm{q}}}(\infty)
\end{array}\right] .
$$

It is interesting to point out the analogy between $\widetilde{Z}_{\dot{\mathrm{w}}}$ and $A_{33}, \widetilde{Z}_{\dot{\mathrm{q}}}$ and $A_{35}, \widetilde{M}_{\dot{\mathrm{w}}}$ and $A_{35}$ and $\widetilde{M}_{\dot{\mathrm{q}}}$ and $A_{55}$, namely the acceleration oscillatory coefficients in the body axes and the added mass coefficients in the equilibrium axes. The relationships between these coefficients are given by Bailey et al [8].

The functions $f_{\mathrm{w}}$ and $f_{\mathrm{q}}$ physically represent the forces (for heave) and moments (for pitch) acting on the ship. These can be written as:

$$
f_{\mathrm{w}}=Z_{\tau}+Z_{\alpha \mathrm{r}}+\widetilde{Z}_{\mathrm{w}}(\infty) w+\widetilde{Z}_{\mathrm{q}}(\infty) q+m q U
$$

for heave, and

$$
f_{\mathrm{q}}=M_{\tau}+M_{\mathrm{\alpha r}}+\tilde{M}_{\mathrm{w}}(\infty) w+\tilde{M}_{\mathrm{q}}(\infty) q
$$

for pitch. In these equations the terms $\widetilde{Z}_{\mathrm{w}}, \widetilde{Z}_{\mathrm{q}}, \widetilde{M}_{\mathrm{w}}$ and $\tilde{M}_{\mathrm{q}}$ contain the infinite values of the velocity oscillatory coefficients. These are analogous to the damping coefficients $B_{33}, B_{35}, B_{53}$ and $B_{55}$ used in the conventional seakeeping analysis and relationships between them and the oscillatory coefficients are given by Bailey et al [8]. The main difference is that the former are defined with reference to the body axes whereas the latter are, by definition, expressed in the equilibrium axes. In equations 8 and $9 Z_{\tau}$ and $M_{\tau}$ are the forces and moments due to radiation potentials. These are expressed in terms of convolution integrals, namely:

$Z_{\tau}=\int_{0}^{t} z_{\mathrm{w}}^{*}(\tau) w(t-\tau) d \tau+\int_{0}^{t} z_{\mathrm{q}}^{*}(\tau) q(t-\tau) d \tau$

and

$$
M_{\tau}=\int_{0}^{t} m_{\mathrm{w}}^{*}(\tau) w(t-\tau) d \tau+\int_{0}^{t} m_{\mathrm{q}}^{*}(\tau) q(t-\tau) d \tau
$$

In equations 10 and $11 z_{\mathrm{w}}{ }^{*}$ and $z_{\mathrm{q}}{ }^{*}$ represent the heaveheave and heave-pitch IRFs, whereas $m_{\mathrm{w}}{ }^{*}$ and $m_{\mathrm{q}}{ }^{*}$ are the pitch-heave and pitch-pitch IRFs. In the present work these IRFs are obtained from the frequency domain hydrodynamic data (i.e. hydrodynamic damping coefficients) through the use of discrete Fourier transforms. Thus, it can be seen that these hydrodynamic forces and moments are with reference to the mean wetted surface.

The terms $Z_{\alpha \mathrm{r}}$ and $M_{\mathrm{\alpha r}}$ in equations 8 and 9 account for the wave disturbance (incident and diffraction) and restoring actions. The Froude-Krylov (incident wave) and restoring actions are evaluated over the instantaneous wetted surface. This requires discretisation (i.e. panelling) of the entire surface of the ship, up to the main deck, and identification of the instantaneous attitude of the ship with respect to the incident wave. Subsequently the pressures over the instantaneous underwater portion of the hull are summed up to provide relevant forces and moments. On the other hand the diffraction actions are evaluated in a manner similar to equation 10 or 11 . That is to say the frequency domain diffraction force (or moment) provides an IRF, through discrete Fourier transform, and the diffraction actions (with respect to the mean wetted surface) are expressed as convolution integrals [9].

Finally, the time domain evaluation of the vessel's motions is carried out using a fourth order Runge-Kutta method in which the velocities are calculated for a set of time steps of a fixed increment. At the start of a simulation, the calm water equilibrium position of the vessel is determined. The subsequent motions are then calculated with reference to this initial position [9].

\section{RESULTS}

The methods outlined in sections 2 and 3 are applied to the prediction of the motions of the S175 container ship travelling in regular head waves. The main particulars of the containership are shown in Table 1 . The body plan of the S175 container ship is shown in Figure1.

The first step is to identify a suitable idealization of the mean wetted surface, in terms of obtaining a converged solution with the number of panels used. To this end panel numbers between 288 and 2358 were used to idealise the mean wetted surface, ensuring an adequate panel aspect ratio of 2:1 [15]. The crudest and finest mean wetted surface idealizations are shown in Figures $2(a, b)$ respectively. As an example the variation of heave and pitch non-dimensional damping coefficients, obtained from the pulsating source method, for different mean wetted surface idealizations are shown in Figure 3 for $F n=0.2$. Examining the dependence of all the hydrodynamic coefficients on number of panels used, it was concluded that use of 288 panels showed large differences compared to other idealizations. Furthermore 
the results using 1058, 1450 and 2358 panels showed negligible differences, indicating that convergence has been achieved. This is confirmed by the heave (heave/wave amplitude) and pitch (pitch/maximum wave slope) RAOs shown in Figure 4 for $F n=0.2$. Based on this results the mesh with 1058 panels was selected to perform the remainder of the linear seakeeping analysis.

In addition, for the mesh up to the deck used for the partly non linear method, the panel size was held as close as possible to the panel size of the mean wetted surface idealization with 1058 panels. This resulted in a mesh of the S175 model up to deck line with 2880 panels, shown in Figure 2(c). It should be noted that although the range of encounter frequencies shown in Figure 3 is limited, the hydrodynamic coefficients were evaluated for a larger range of encounter frequencies in order to obtain an accurate discrete Fourier transform to obtain the IRFs [9].

There are two sets of results. The first set comprises variation of heave and pitch RAOs with encounter frequency for a range of Froude numbers; $F n=0.2,0.25$ and 0.275 when using the pulsating source and partly nonlinear methods, $F n=0.2$ and 0.275 when using the translating, pulsating source method. In the case of the partly nonlinear analysis three wave amplitudes are investigated, i.e. $\mathrm{a}=1,3$ and $5 \mathrm{~m}$. These results are shown in Figures 5, 6 and 7 for Froude numbers $F n=0.2,0.25$ and 0.275 , respectively. It should be noted that the pitch RAO is in the form of pitch amplitude (rads)/wave amplitude. The reason for this choice is that the numerically predicted pitch RAO, defined as $\mathrm{pitch} /$ maximum wave slope, tends to large values when the frequency tends towards zero due to the division of two relatively small numbers. This can be clearly seen in Figure 4.

First let us focus on the trends of the predictions obtained by the partly nonlinear method. The differences in predicted RAOs due to different wave amplitudes become notable in the vicinities of the peaks. For both heave and pitch RAOs and all Froude numbers investigated a decrease is observed, in general, in the RAO with increasing wave amplitude. A notable exception to this trend relates to the heave RAOs at $\omega_{\mathrm{e}}=1 \mathrm{rad} / \mathrm{s}$ for all $\mathrm{Fn}$ values. In fact for $\mathrm{Fn}=0.275$ the heave RAO predicted in $5 \mathrm{~m}$ amplitude waves is nearly zero and out of line with the general trends observed. It should be noted that a wave amplitude $\mathrm{a}=5 \mathrm{~m}$ corresponds to a rather steep wave, i.e. wave steepness values ka in excess of 0.12 , as can be seen from Table 2. The predicted pitch RAO, at the same frequency also shows mixed trends with the pitch RAO for $\mathrm{a}=3 \mathrm{~m}$ being larger or same as that for $\mathrm{a}=1 \mathrm{~m}$, at $\mathrm{Fn}=0.25$ and 0.275 , respectively. The rate of decreases in the heave RAOs with increasing wave amplitude remains more or less unchanged with increasing Froude number. On the other hand this rate increases with increasing Froude number for the pitch RAOs, as can be seen by comparing Figures 5,6 and 7. In general the rate of change in the RAOs is larger from $1 \mathrm{~m}$ to $3 \mathrm{~m}$ wave amplitude and smaller from $3 \mathrm{~m}$ to $5 \mathrm{~m}$ wave amplitude. This requires further investigation and validation to establish whether it is a real trend or due to the partly nonlinear method reaching the limits of its validity.

It is important to compare the differences between the RAOs predicted by the linear pulsating source method and the partly nonlinear method. For the lowest of forward speeds $(\mathrm{Fn}=0.2)$ the linear heave RAO is smaller than the partly nonlinear prediction for $\mathrm{a}=1 \mathrm{~m}$. The heave RAOs predicted by the partly nonlinear method for $\mathrm{a}=1 \mathrm{~m}$ are the same and smaller than the linear predictions for $F n=0.25$ and 0.275 , respectively. The trend observed for $\mathrm{Fn}=0.2$ is contrary to expectations of the behaviour of nonlinear methods. As this effect seems to be more pronounced at low speeds it may be linked to the differences in the hydrostatic restoring actions between the linear method based on the mean wetted surface and the partly nonlinear method using the instantaneous wetted surface. Thus it may be possible to argue that at higher Froude numbers the hydrodynamic actions have a more pronounced effect, hence decreasing the influence of the differences in the hydrostatic restoring coefficient. On the other hand the pitch RAOs predicted by the pulsating source method are, in general, larger than the partly nonlinear predictions for all Froude numbers. Nevertheless for $F n=0.275$ the pitch RAO predicted by the partly nonlinear method for $\mathrm{a}=1 \mathrm{~m}$ is very close to the linear (pulsating source) prediction. The aforementioned reasoning on the differences of the hydrostatic coefficients may also explain the trends observed when comparing linear and partly nonlinear pitch predictions. This issue requires further investigation.

The RAO predictions obtained from the translating pulsating source method are shown in Figures 5 and 7, for $F n=0.2$ and 0.275 , respectively. The heave RAOs predicted by the pulsating and the translating, pulsating source methods are comparable for both Froude numbers. At the highest speed $(\mathrm{Fn}=0.275)$ the pitch RAOs predicted by the translating, pulsating source are much higher than the pulsating source predictions, peak values approximately 3.5 times higher. Examining the pitch damping coefficient $B_{55}$, shown in Figure 3, it can be seen that the value predicted by the translating, pulsating source method is much lower than that of the pulsating source method in the frequency range where the pitch RAO peaks. Similar trends between pulsating and translating, pulsating source predictions, at relatively high Froude numbers were observed for a NPL hull form [16]. This is an important issue in terms of the applicability of the translating, pulsating source method, especially at higher speeds. The influence of the steady flow and its effect on the body boundary conditions (rather than using the simplified conditions given by Equation 3) may provide an explanation of the differences observed [14].

The second set of results provides heave and pitch RAOs for three wave to ship length ratios, namely $\lambda / \mathrm{L}=1.0,1.2$, 
1.4, a range of wave steepness values ka and two Froude numbers $F n=0.2$ and 0.275 . These are shown in Figures 8 and 9 , respectively. The results predicted by the partly nonlinear method are compared with the experimental measurements presented by O'Dea et al [10]. The corresponding linear pulsating source predictions are also shown, in the form of constant lines for each $\lambda / \mathrm{L}$ value. The relationships between various wave properties for this second set of results are shown in Table 2 .

The basic trend displayed by the experimental results, namely a decrease in RAOs with increasing wave steepness $\mathrm{ka}$ is observed in all predictions by the partly nonlinear method, except for $\lambda / \mathrm{L}=1.0$ and $\mathrm{Fn}=0.275$ where the predictions show a small increase with increasing ka values. It can be seen from Table 2 that this corresponds to $\omega_{\mathrm{e}}=1$ in Figure 7 and was discussed above. It is interesting to note that both heave and pitch RAOs in Figure 7 show a decrease when $a=5 m$, tying up with the experimental trends for ka values in excess of 0.12 . Heave RAOs predicted by the partly nonlinear method are higher than the experimental measurements, as well as the linear predictions by the pulsating source method, for $\mathrm{Fn}=0.2$. Pitch RAOs predicted by the partly nonlinear method are a little higher than the experimental measurements, and the pulsating source prediction, for $\lambda / \mathrm{L}=1$ and $F n=0.2$. For the same $F n=0.2$, pitch RAOs predicted by the partly nonlinear method are lower than the experimental measurements, and closer to these measurements than the linear prediction, for $\lambda / \mathrm{L}=1.2$ and 1.4. Heave RAOs predicted by the partly nonlinear method are closer to the experimental measurements, though a little higher, than the linear pulsating source prediction for $\lambda / \mathrm{L}=1.2$ and 1.4 and $F n=0.275$. Pitch RAOs predicted by the partly nonlinear method for $F n=0.275$ and for $\lambda / L=1.2$ and 1.4 are close to each other, as are the linear predictions, and close to the experimental measurements for $\lambda / L=1.4$, but higher than the measurements for $\lambda / \mathrm{L}=1.2$. Overall it can be said that the partly nonlinear method offers improvement in predictions with reference to trends with increasing wave steepness. The quantitative agreement, based on the limited set of measurements used here, is reasonably good, although it can be patchy on occasion.

\section{INVESTIGATING NONLINEAR MOTIONS}

The results discussed in section 4 demonstrate the need for further comparison studies with experimental measurements for a range of ship types and operational conditions. Such tests will ascertain the range of validity of partly nonlinear methods. Furthermore, they emphasize the need for the development of methods that allow for nonlinearities in the radiation and diffraction potentials.

Fully nonlinear methods are, in theory, capable of accounting for the non linear effects in potential flow. Developments in this direction mainly rely on the mixed Eulerian Lagrangian (MEL) description of the flow. The foundation of the MEL method was established originally to simulate steep waves in two dimensions by LonguetHiggins \& Cokelet [17]. The main idea behind the MEL scheme is to approximate the nonlinear solution by solving a linear problem at each time step, the so called initial boundary value problem. Thus, at each time step, the simulation can be split in two main steps. During the first step (Eulerian phase), given a set of suitable boundary conditions (Dirichlet, Neumann or sometimes both conditions) the conventional boundary integral equations (BIE) are solved and the velocity potential and its velocity field are calculated. In the second step (Lagrangian phase), the position and the velocity potential of the free surface are updated explicitly by the velocity field calculated in the Eulerian phase. The process is then repeated for the next time step.

MEL schemes, because of their flexibility, have been applied to a broad range of hydrodynamic problems. For instance, Beck et al [18] used a desingularised boundary element solver, based on the indirect Rankine panel method, to carry out seakeeping analysis, in two and three dimensions, of simple hull forms. Liu et al [19] applied a higher order direct Rankine panel method to simulate overturning waves in three dimensions and to model the hydrodynamic resistance problem of a Series 60 hull form. Unfortunately, although relatively simple in theory, MEL implementations bring their own problems especially in the presence of the floating body, due to the mixed nature of the boundary value problem. Bai \& Eatock Taylor [20] discuss a range of problems when carrying out simulations of motions of flared floating structures in the context of non linear potential flow theory. These include the double node boundary condition and its influence on the derivatives of the potentials and the decoupling of the diffraction problem. In fact, problems encountered in the implementation of MEL have been limiting its application to more realistic hull forms and more realistic problems [21].

It is also worth pointing out that due to its Lagrangian feature MEL schemes are interface tracking schemes. This is in contrast to most of the CFD RANS (Reynolds Averaged Navier Stokes equations) schemes which, when applied to the seakeeping problem, model the free surface behaviour based on an interface capturing scheme (Eulerian scheme). There are a number of different methods for capturing the interface evolution in an Eulerian framework. Most of them are based on convecting the volume fraction of water, so called volume of fluid method, VoF [e.g. 22]. Another possibility is to use level set theory. Accordingly, once the velocity field is given, the free surface is convected as a level set function [e.g. 23, 24]. Applications of interface capturing methods to the fluid-structure interactions problem, although computationally more expensive than MEL (i.e. interface tracking) schemes, has made considerable progress during recent years [e.g. 22, 25]. An interesting approach, which is currently being investigated, is to try and combine a level set interface capturing technique with a non linear potential flow 
solver. In this approach, at each time step, the mixed boundary value problem is solved providing the velocity field. A level set approach is then used to convect the free surface for the computed velocity field. The process is then repeated at each time step. The main advantage of this approach is that the domain can be described by a signed distance function which considerably simplifies the mesh generation procedure [24]. An obvious disadvantage is the fact that an Eulerian grid needs to be introduced, which increases the size of problem.

\section{CONCLUSIONS}

An investigation has been carried out comparing predictions obtained from three-dimensional linear, pulsating and translating, pulsating source, and partly nonlinear methods, together with comparisons with available experimental measurements. The S175 containership, travelling regular head waves at a range of Froude numbers and wave amplitudes, was used as an example for this investigation.

Based on this limited investigation it can be concluded that the partly nonlinear method offers, in general, improvements in predicting heave and pitch RAOs. Nevertheless, more comparisons with experimental measurements are necessary in order to establish the range of validity of this method.

In the case of linear methods, the pulsating source method produces better predictions, by comparison to the translating, pulsating source method at reasonably high Froude numbers. The influence of steady flow on the translating, pulsating source method needs further investigation.

The quality of the agreement with experimental measurements tends to emphasize the need for development of nonlinear methods, still within the potential flow domain, accounting for nonlinearities in radiation and diffraction potentials. The difficulties in obtaining such a numerically stable method were discussed.

\section{ACKNOWLEDGEMENTS}

The authors acknowledge the support from the Lloyd's Register Strategic Research Group.

'Lloyd's Register, its affiliates and subsidiaries and their respective officers, employees or agents are, individually and collectively, referred to in this clause as the "Lloyd's Register Group". The Lloyd's Register Group assumes no responsibility and shall not be liable to any person for any loss, damage or expense caused by reliance on the information or advice in this document or howsoever provided, unless that person has signed a contract with the relevant Lloyd's Register Group entity for the provision of this information or advice and that in this case any responsibility or liability is exclusively on the terms and conditions set out in that contract'.

\section{REFERENCES}

1. INGLIS, R.B. \& PRICE, W.G. 'A three dimensional ship motion theory: Comparison between theoretical predictions and experimental data of the hydrodynamic coefficients with forward speed', Transactions of RINA, 124, 141-157, 1982.

2. SCLAVOUNOS, P.D, NAKOS, D.E. \& HUANG, Y. 'Seakeeping and wave induced loads on ships with flare by a Rankine panel method', Proceedings of the $6^{\text {th }}$ International Conference in Numerical Ship Hydrodynamics, Iowa, 1993.

3. INGLIS, R.B. \& PRICE, W.G. 'Calculation of the velocity potential of a translating, pulsating source', Transactions of RINA, 123, 163-175, 1981.

4. INGLIS, R.B. \& PRICE, W.G. 'A three-dimensional ship motion theory: Calculation of wave loading and response with forward speed', Transactions of RINA, 124, 183-192, 1982.

5. ISSC 'Report of Committee I.2 Loads', Proceedings of the $17^{\text {th }}$ International Ships and Offshore Structures Congress, 1, 127-210, Korea, 2009.

6. LIN, W.M \& YUE, D. 'Numerical solutions for large amplitude ship motions in the time domain', Proceedings of the $18^{\text {th }}$ Symposium on Naval Hydrodynamics, 41-66, USA, 1990.

7. LIN, W.M., MEINHOLD, M., SALVESEN, N. \& YUE, D.K.P. 'Large-amplitude motions and wave loads for ship design', Proceedings of the $20^{\text {th }}$ Symposium on Naval Hydrodynamics, 205-226, 1994.

8. BAILEY, P.A., HUDSON, D.A., PRICE, W.G. \& TEMAREL, P. 'A unified mathematical model describing the manoeuvring of a ship travelling in a seaway', Transactions of RINA, 140,131-149, 1998.

9. BALLARD, E. J., HUDSON, D. A., PRICE, W.G. \& TEMAREL, P. 'Time domain simulations of symmetric ship motions in waves', Transactions of RINA, 145, 89108,2003

10. O'DEA, J., POWERS, E. \& ZSELECSKY, J. 'Theoretical and experimental study of the non linearities in vertical plane ship motions', Proceedings of the $19^{\text {th }}$ Symposium on Naval Hydrodynamics, 73-91, 1992.

11. NEWMAN, J.N. 'Marine Hydrodynamics', MIT Press, 1977. 
12. OGILVIE, F. \& TUCK, E.O. 'A rational strip theory for ship motions', University of Michigan, Technical Report 013, 1969.

13. INGLIS, R.B. \& PRICE, W.G. 'The influence of speed dependent boundary conditions in three dimensional ship motion problems', International Shipbuilding Progress, 28, 22-29, 1981.

14. AHMED, T.M., HUDSON, D.A. \& TEMAREL, P. 'Incorporation of steady flow effects in linear threedimensional seakeeping predictions for high speed hulls', Proceedings of the $9^{\text {th }}$ International Symposium on Practical Design of Ships and Other Floating Structures, 1, 496-503, 2004.

15. BAILEY, P.A., HUDSON, D.A., PRICE, W.G. \& TEMAREL, P. 'A simple yet rational approach to the panelling of hull surfaces', Transactions of RINA, 144, 4961, 2002.

16. BAILEY, P.A., HUDSON, D.A., PRICE, W.G. \& TEMAREL, P. 'Theoretical and experimental validation of the seakeeping characteristics of high speed mono- and multi-hulled vessels', Proceedings of the $5^{\text {th }}$ International Conference on Fast Sea Transportation, Seattle, Washington, USA, 1999.

17. LONGUET-HIGGINS, M.S. \& COKELET, C.D. 'The deformation of steep surface waves on water I: A numerical method of computation', Proceedings of the Royal Society London, A350, 1-26, 1976.

18. BECK, R.F., CAO, Y., SCORPIO, S. \& SCHULTZ, W. 'Nonlinear ship motion computations using the desingularized method', Proceedings of the $20^{\text {th }}$ Symposium on Naval Hydrodynamics, USA, 227-247, 1994.

19. LIU, Y., XUE, M. \& YUE, D.K. P. 'Computation of fully nonlinear three-dimensional wave-wave and wavebody interactions. Part 2 Nonlinear waves and forces on a body', Journal of Fluid Mechanics, 438, 41-65, 2001.

20. BAI, W. \& EATOCK TAYLOR, R. 'Fully nonlinear simulation of wave interaction with fixed and floating flared structures', Journal of Ocean Engineering, 36, 223236, 2009.

21. Diken, Ö., DU, S.X., HUDSON, D.A. \& TEMAREL, P. 'Calculation of steady-state ship wave patterns using a general Rankine source method', Proceedings of the $14^{\text {th }}$ International Offshore and Polar Engineering Conference, 447-454, 2004.

22. VAZ, G., JAOUEN, F. \& HOEKSTRA, M. 'Free surface viscous flow computations. Validation of URANS code FRESCO', Proceedings of the $28^{\text {th }}$ International Conference on Ocean, Offshore and Arctic Engineering, 2009.
23. DI MASCIO, A., BROGLIA, R. \& MUSCARI, R. 'On the application of the single-phase level set method to naval hydrodynamic flows', Journal of Computers and Fluids, 36, 868-886, 2007.

24. CHEN, Y.G. \& PRICE, W.G. 'Numerical simulation of liquid sloshing in a partially filled container with inclusion of compressibility effects', Physics of Fluids, 21, 112105, 2009.

25. CHEN, Y.G., PRICE, W.G. \& TEMAREL, P. 'An anti-diffusive VoF method for computational modelling of sloshing in an LNG tank', Proceedings of the $11^{\text {th }}$ International Symposium on Practical Design of Ships and Other Floating Structures, Brasil, 1, 5-15, 2010.

\section{AUTHORS BIOGRAPHY}

Alberto Chapchap completed his MSc course in Naval Architecture at the University of Sao Paolo, Brasil, in 2009. He is currently a PhD student at the University of Southampton.

Tamer Ahmed obtained his $\mathrm{PhD}$ degree in 2004 at the University of Southampton. He worked at the same University as a post-doctoral Research Fellow until 2010. His expertise is on the area of ship hydrodynamics, focusing on seakeeping and parametric roll. He currently works at the Department of Naval Architecture and Marine Engineering, Alexandria University, Egypt.

Dominic Hudson is a Senior Lecturer at the University of Southampton with research interests in all aspects of ship hydrodynamics, in particular the motions of high speed craft in waves and ship design for energy efficiency. He is currently course coordinator for the Ship Science degree programmes at the University.

Pandeli Temarel is Professor of Hydroelasticity at the School of Engineering Sciences, University of Southampton. He has extensive experience of fluidstructure interaction problems and, in particular, hydrodynamics, seakeeping, structural dynamics, hydroelasticity, manoeuvring and stability

Spyros Hirdaris CEng MRINA holds a $\mathrm{PhD}$ from the University of Southampton, UK. He has been working for the Lloyd's Register Innovation, Strategic Research and Product Development Departments since 2004. To date his work has involved development of Design Procedures for Marine Structures and Hydrodynamic Loads, technology investigations as well as coordination of $R \& D$ activities. He is currently responsible for the Lloyd's Register Strategic Research programme on Hydrodynamics in association with the Universities of Southampton, Strathclyde and Newcastle in the UK. He is the Chair of the ISSC Committee I. 2 on Loads. 


\begin{tabular}{|l|l|}
\hline Length between perpendiculars $(\mathrm{m})$ & 175 \\
\hline Beam $(\mathrm{m})$ & 25.4 \\
\hline Depth $(\mathrm{m})$ & 15.4 \\
\hline Draught $(\mathrm{m})$ & 9.5 \\
\hline Displacement (tonnes) & 24860 \\
\hline Pitch radius of gyration $(\mathrm{m})$ & 42.1 \\
\hline
\end{tabular}

Table 1: Main particulars of the S175 container ship

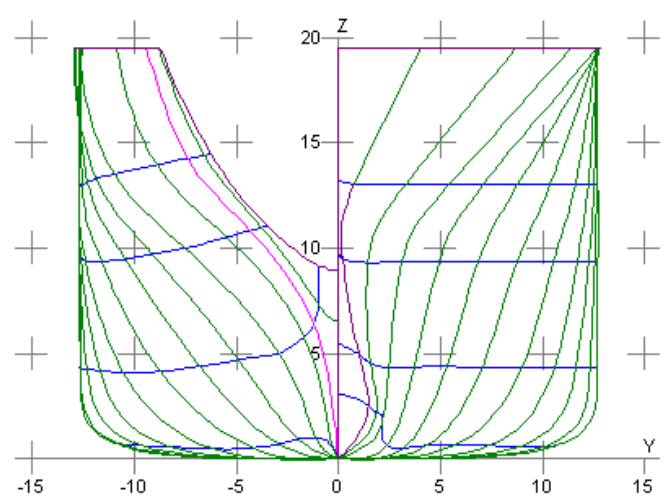

Figure 1: Body plan of the S175 container ship
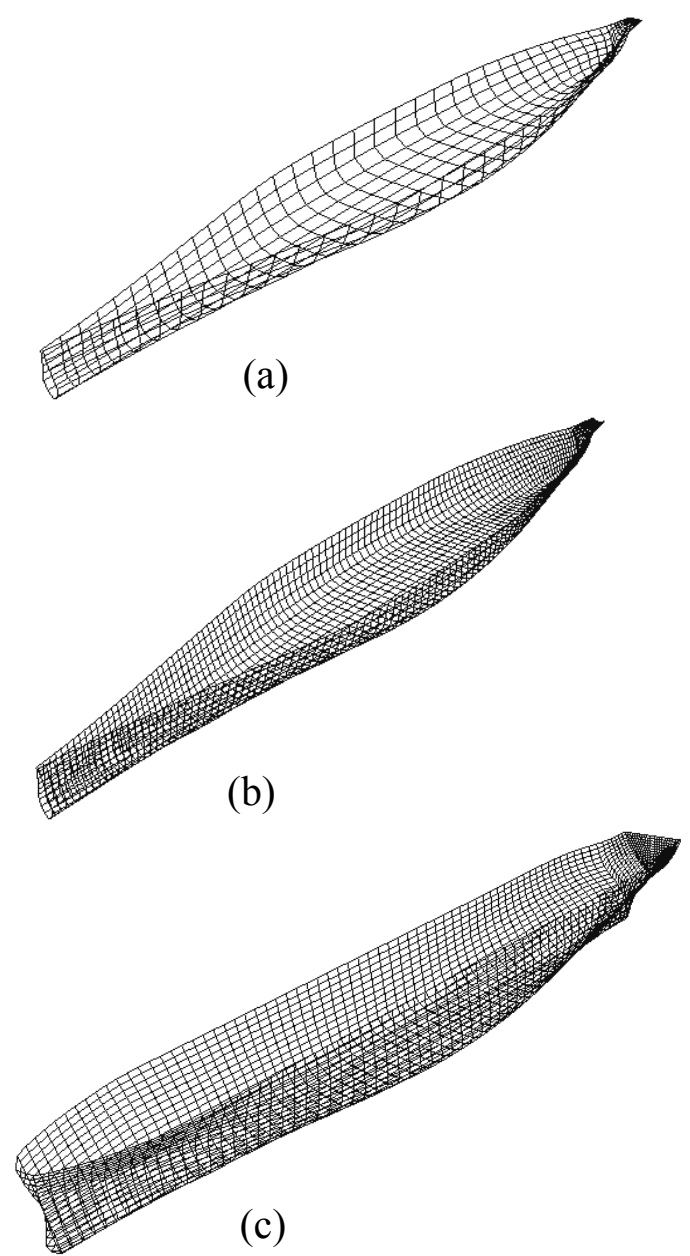

Figure 2: Idealization of the surface of the S175 containership; (a) 588 and (b) 2358 panel idealizations of mean wetted surface, (c) 2880 panel idealization of the whole surface up to deck for the partly nonlinear method

\begin{tabular}{|c|c|c|c|}
\hline & $\lambda / \mathrm{L}=1.4$ & $\lambda / \mathrm{L}=1.2$ & $\lambda / \mathrm{L}=1.0$ \\
\hline$\omega(\mathrm{rad} / \mathrm{s})$ & 0.500 & 0.54 & 0.59 \\
\hline $\begin{array}{c}\omega_{\mathrm{e}}(\mathrm{rad} / \mathrm{s}), \\
\mathrm{Fn}=0.20\end{array}$ & 0.71 & 0.79 & 0.89 \\
\hline $\begin{array}{c}\omega_{\mathrm{e}}(\mathrm{rad} / \mathrm{s}), \\
\mathrm{Fn}=0.275\end{array}$ & 0.79 & 0.88 & 1.01 \\
\hline $\mathrm{k}(1 / \mathrm{m})$ & 0.025 & 0.030 & 0.036 \\
\hline $\mathrm{ka}$ & \multicolumn{3}{|c|}{$\mathrm{a}(\mathrm{m})$} \\
\hline 0.01 & 0.39 & 0.34 & 0.28 \\
\hline 0.04 & 1.56 & 1.34 & 1.12 \\
\hline 0.08 & 3.12 & 2.68 & 2.23 \\
\hline 0.12 & 4.68 & 4.02 & 3.35 \\
\hline
\end{tabular}

Table 2: Relationship between wave length, wave frequency, wave amplitude and wave slope; encounter frequency in regular head waves
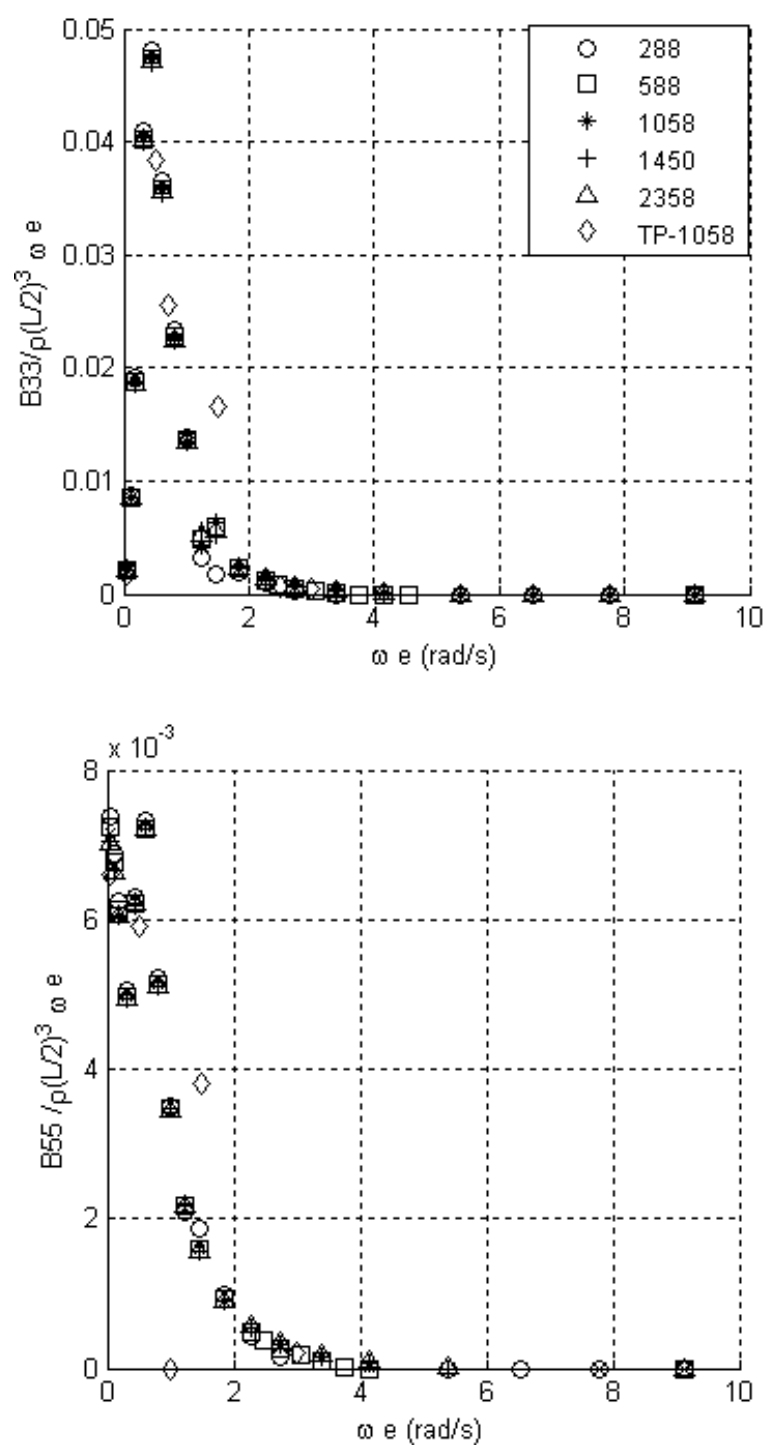

Figure 3: Non-dimensional heave and pitch damping coefficients $(\mathrm{Fn}=0.2)$ obtained using the pulsating source (various panel numbers on mean wetted surface) and the translating, pulsating source (1058 panels on mean wetted surface) 

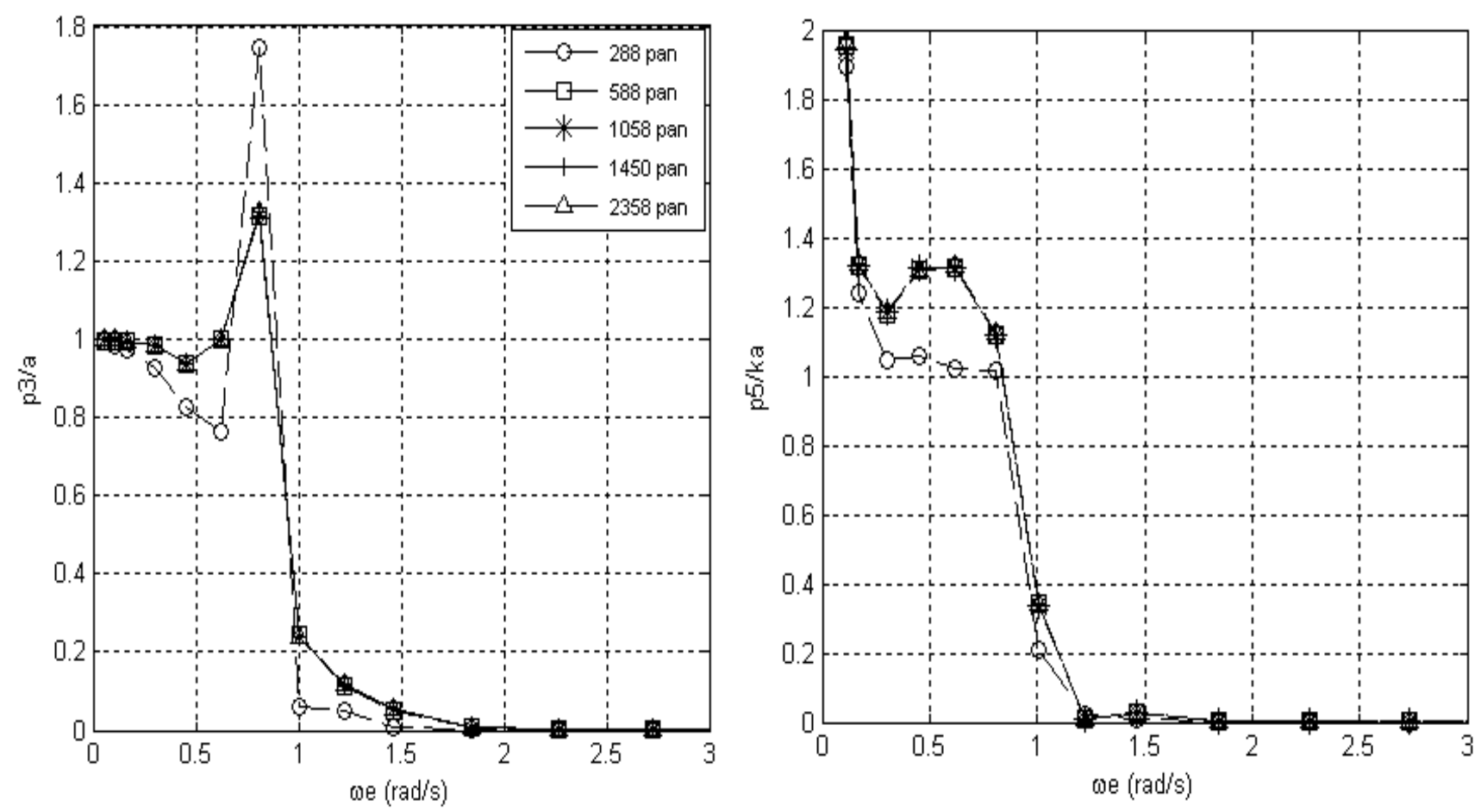

Figure 4: Illustration of convergence for the heave and pitch RAOs, with various panel numbers on the mean wetted surface, obtained using the pulsating source method for the S175 containership travelling in head regular waves, Fn=0.2
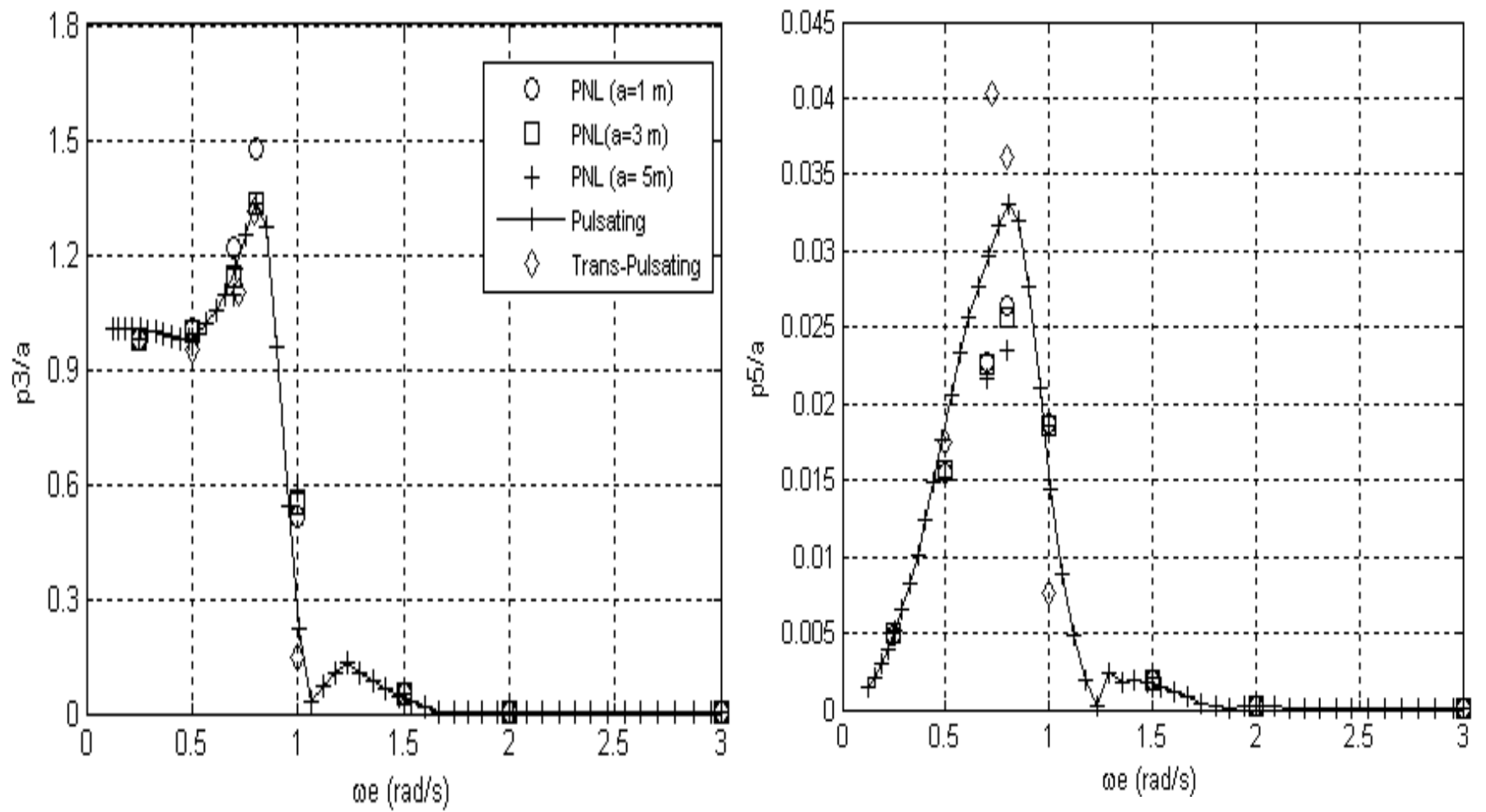

Figure 5: Heave and pitch transfer functions for the S175 containership travelling in head regular waves, Fn=0.2; comparison of linear (pulsating and translating, pulsating source) and partly nonlinear (PNL) methods 

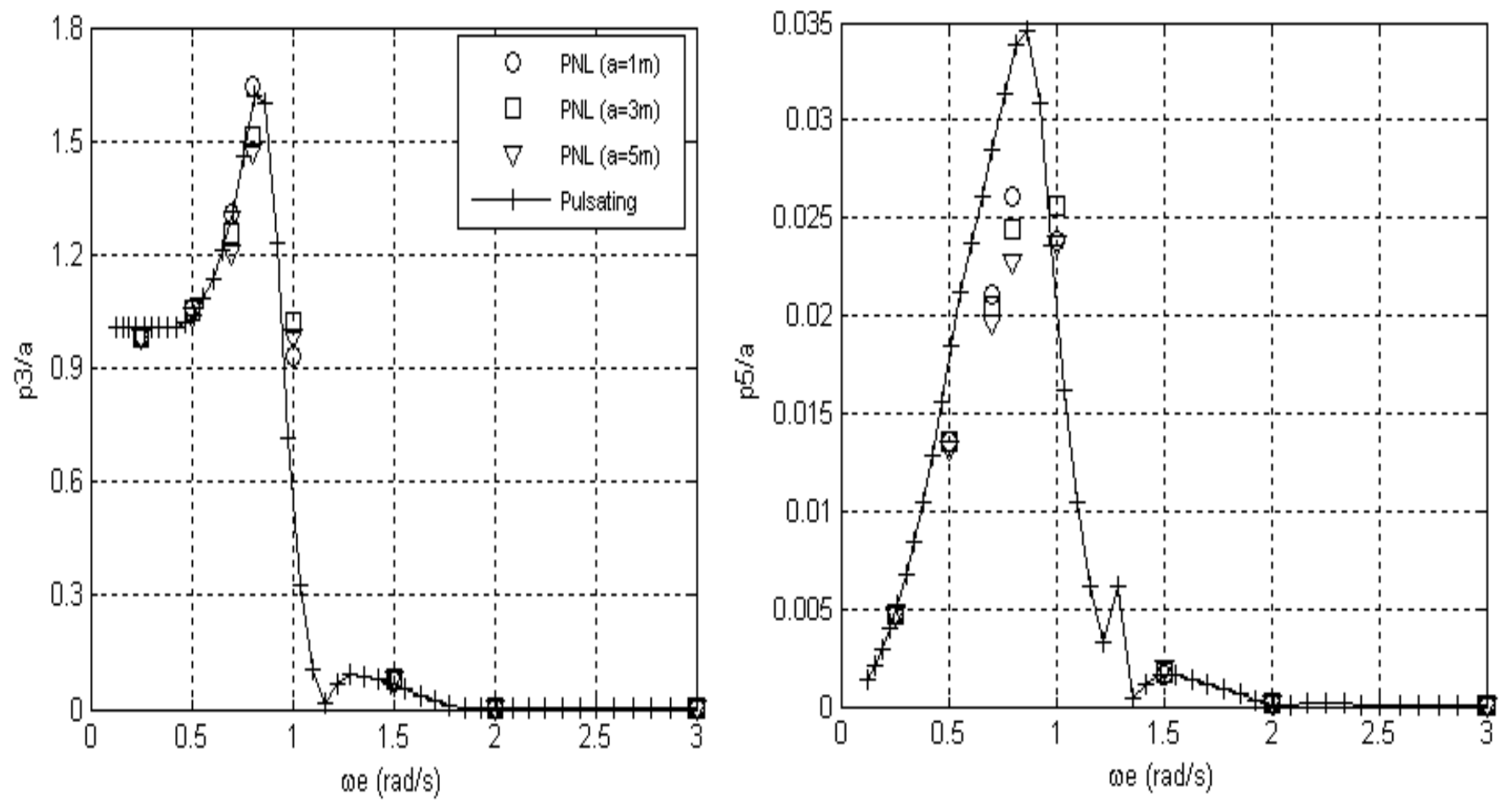

Figure 6: Heave and pitch transfer functions for the S175 containership travelling in head regular waves, Fn=0.25; comparison of linear (pulsating source) and partly nonlinear (PNL) methods
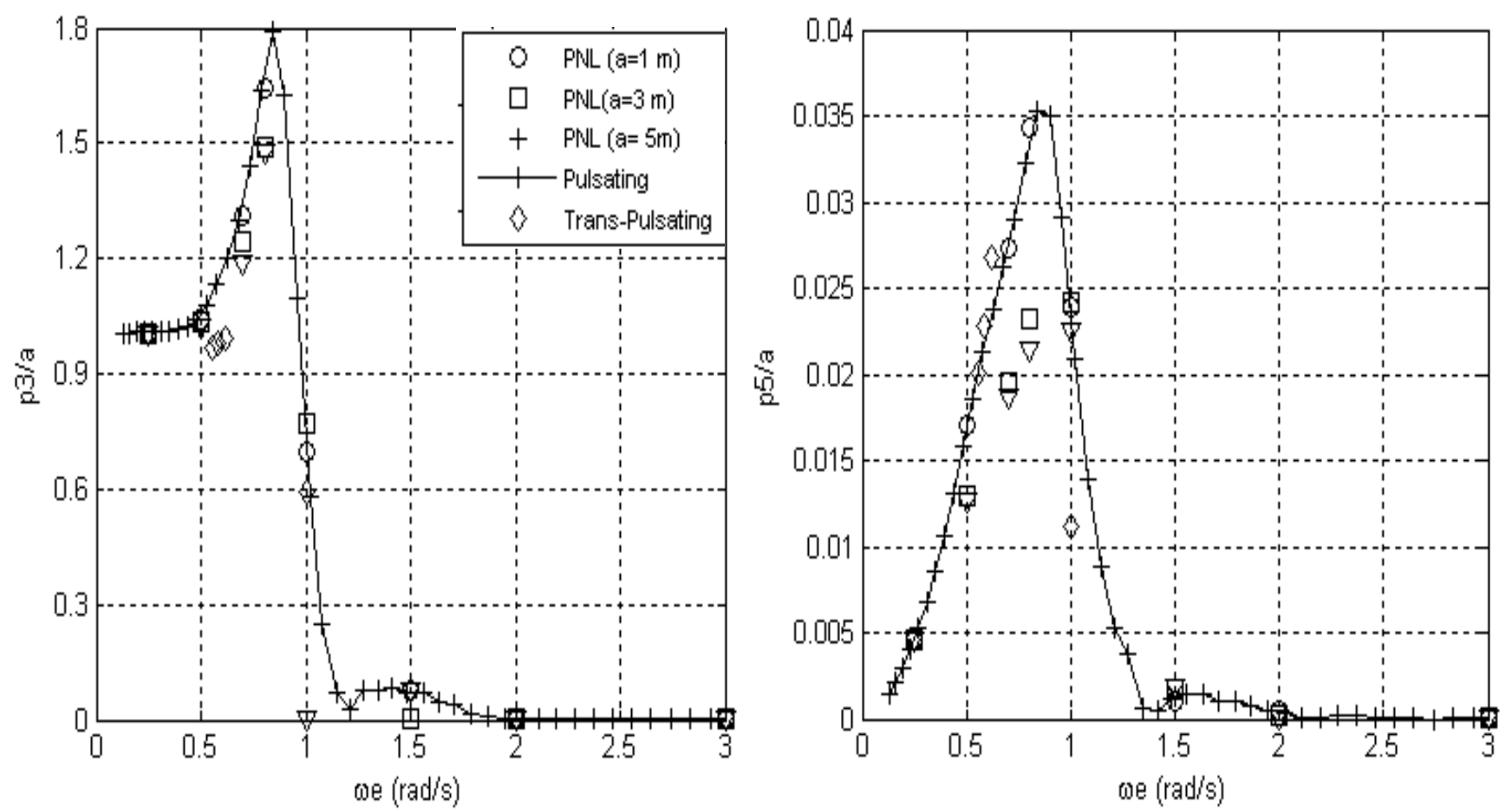

Figure 7: Heave and pitch transfer functions for the S175 containership travelling in head regular waves, Fn=0.275; comparison of linear (pulsating and translating, pulsating source) and partly nonlinear (PNL) methods 

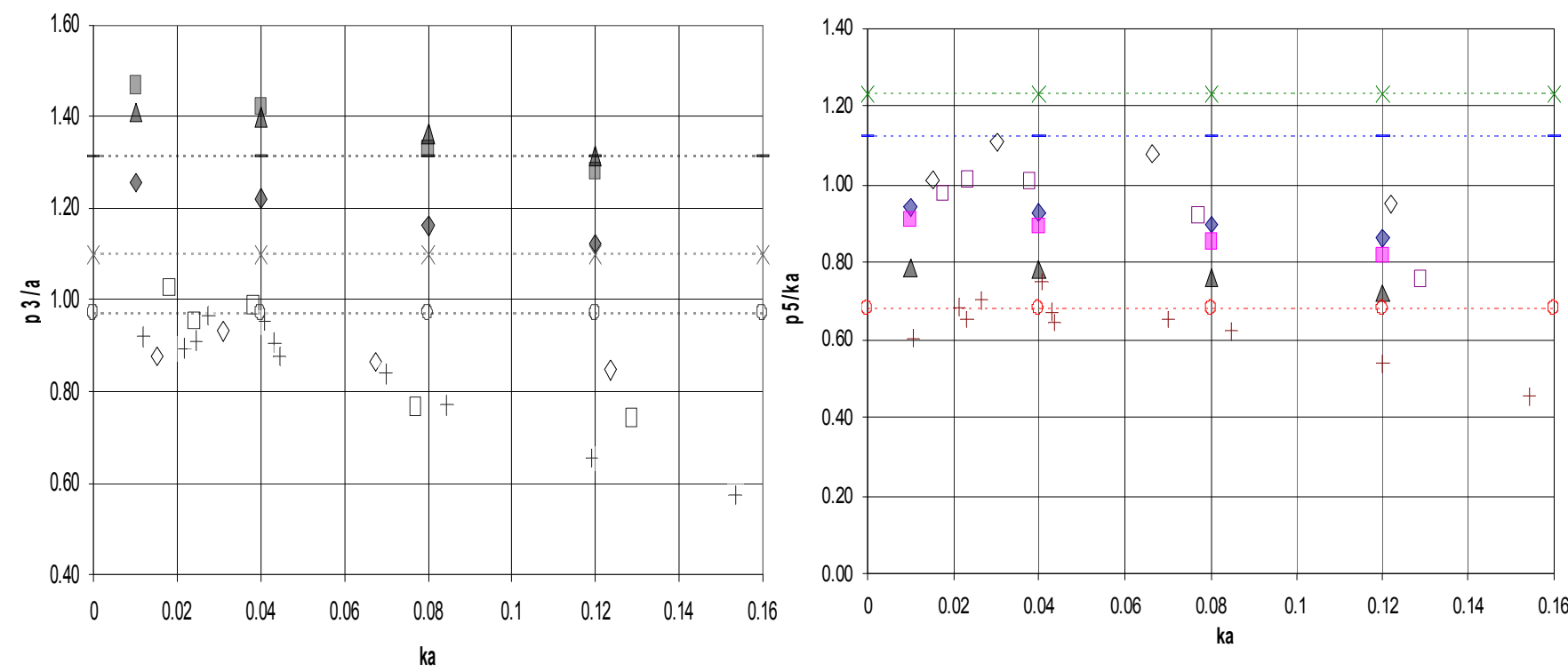

\begin{tabular}{|c|c|c|}
\hline$\lambda / L=1.4$ & $\square \quad \lambda / L=1.2$ & $\Delta \quad \lambda / L=1.0$ \\
\hline$\diamond \quad \exp N / L=1.4$ & $\exp \lambda / L=1.2$ & $+\quad \exp N / L=1.0$ \\
\hline$\cdots{ }_{-L}$ Linear $\lambda / L=1.4$ & $\ldots$. L Linear $\lambda / L=1.2$ & $\ldots \ldots$ Linear $\lambda / L=1.0$ \\
\hline
\end{tabular}

Figure 8: Comparison of numerical (partly nonlinear and linear, pulsating source) and experimental heave and pitch RAOs for the containership travelling in head regular waves, $\mathrm{Fn}=0.2$
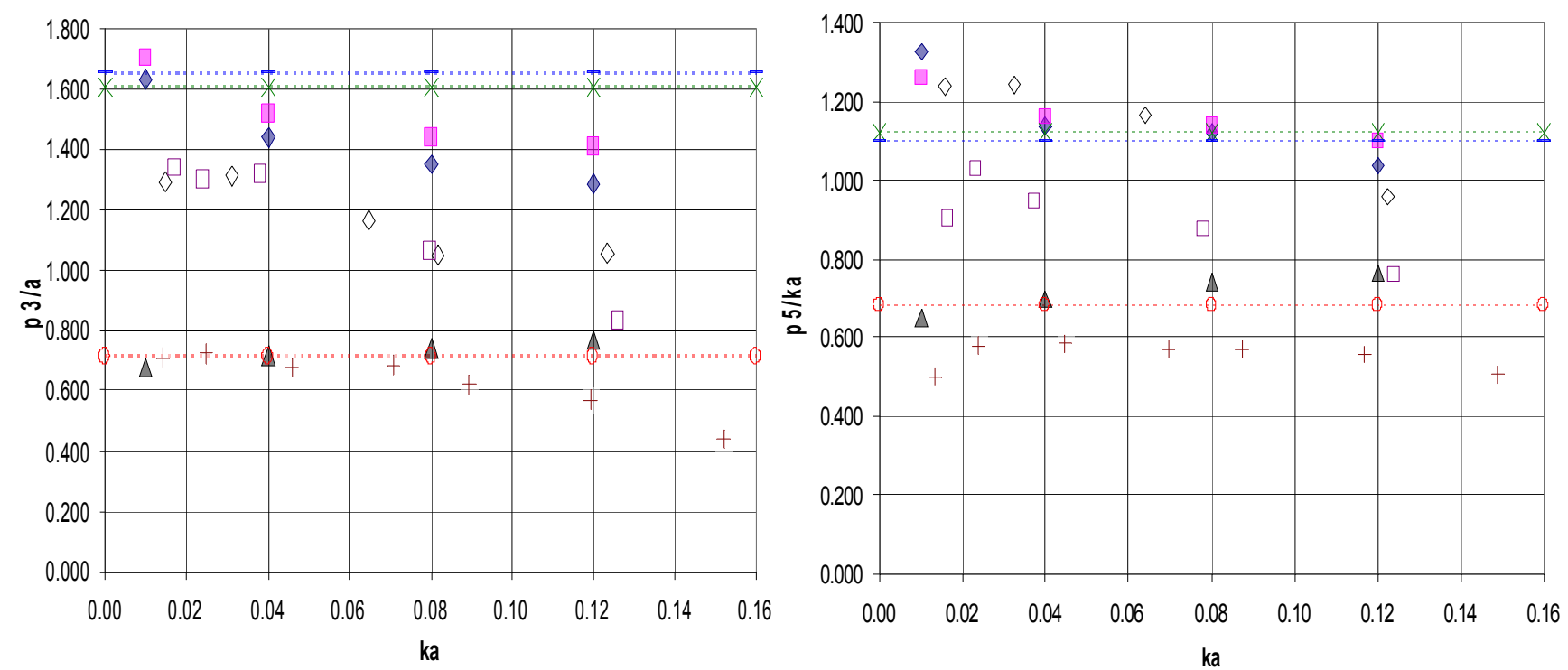

Figure 9: Comparison of numerical (partly nonlinear and linear, pulsating source) and experimental heave and pitch RAOs for the containership travelling in head regular waves, $\mathrm{Fn}=0.275$ 